\title{
Moyamoya disease in the midwestern United States
}

Nicholas M. Wetjen, B.S., P. Charles Garell, M.D., Nicholas V. Stence, and Christopher M. Loftus, M.D.

Division of Neurosurgery, University of Iowa Hospitals and Clinics, College of Arts and Sciences, University of Iowa, Iowa City, Iowa; and Department of Neurosurgery, University of Oklahoma Health Sciences Center, Oklahoma City, Oklahoma

There have been few investigations of moyamoya disease in the United States and no systematic description of the management practices or outcome from this population. The authors reviewed their experience with this disease to gain a better understanding and improve the treatment of patients with moyamoya disease in the United States. Over a 25-year period 30 patients with moyamoya disease have been treated at the University of Iowa. The cases were divided into patients who had classic, probable, and akin moyamoya disease.

Results indicated that there was a bimodal age distribution and a female predominance of cases. In estimating the referral pattern of our institution, the authors determined that there were greater numbers of epidemiological characteristics than previously anticipated. Patients were treated either surgically or nonsurgically, and different management strategies were utilized in each of the major groups: superficial temporal artery to middle cerebral artery anastomosis and encephalodurosynangiosis in the surgical group; or antiplatelet, anticoagulation, or nonpharmacological intervention in the nonsurgical group.

The authors conclude that there is a higher prevalence and incidence of moyamoya disease in the United States than previously reported and that there are some clinical characteristics of this disease that differ from the cases reported in southeast Asia. These differences may be due to genetic or environmental factors but can also be partly explained by the lower index of suspicion for this disease and, thus, a delay in or complete absence of the correct diagnosis.

\section{Key Words * moyamoya disease * outcome study * cerebrovascular disease}

The radiographic characteristics of moyamoya disease were first described by Takeuchi and Shimuzu[16] in the late 1950s and by Kudo[6] in the early 1960s. These descriptions were introduced in the English language literature in 1968 by Kudo.[8] The first report of moyamoya disease consistent with the current criteria for diagnosis was reported by Takeuchi[15] in 1961. In the first case series published in Japan by Nishimoto and Takeuchi[10] the authors developed a symptomatological outline of the disease. 
Although cases of moyamoya disease have been reported worldwide, it is believed to be most prevalent in southeast Asia, especially Japan, China, and Korea. Goto and Yonekawa[3] have shown that cases of moyamoya disease can be found throughout the world but at a lower rate of incidence than in southeast Asia. The Japanese Ministry of Health and Welfare formed a research committee in 1977 to track the epidemiological characteristics of the disease. The most recent nationwide survey was undertaken by this committee in 1994, and the total number of treated patients was estimated to be 3900.[17] The reported prevalence and incidence in Japan were 3.16 and 0.35 per 100,000 citizens, respectively. Women slightly outnumbered men by a ratio of 1.8:1. The disease has a bimodal peak of incidence: development of symptoms occurs in the first decade or in the third and fourth decades of life. Although the cause of the disease remains unknown, there appears to be some degree of genetic predisposition because the entity occurs in families $10 \%$ of the time.

Moyamoya disease is a slow, progressive occlusion of the major trunks of the circle of Willis that begins at the siphon of the internal carotid arteries (ICAs). Although the cause remains unknown, there is some evidence to suggest that functional alterations in vascular cells of the arterial wall play a role in the development of a thickened intima. Pathological and clinical features have been characterized as follows: 1) stenosis or occlusion is observed at the terminal portion of the ICA and at the proximal portion of the anterior cerebral artery, middle cerebral artery, or both; and 2) abnormal vascular networks then form and are observed in the vicinity of the occluded anterior circulation bilaterally.[19] Pathological findings typical of moyamoya disease include intimal proliferation with resulting stenosis and/or occlusion around the terminal portion of the intracranial ICA. This is sometimes associated with lipoidal degeneration in the wall, without evidence of chronic inflammation.[19] The main arteries of the circle of Willis are characterized by fibrous thickening of the intima, widening of the internal elastic lamina, and thinning of the tunica media. It is thought that the development of these histological features may be due to aberrant growth factor signals and/or induction from the arterial vessel layers that overlie one another. The occlusion of the arteries observed on angiography and pathological examination results in the development of communicating, collateral networks of arterial vessels around the circle of Willis.

The first case of moyamoya disease at the University of Iowa was treated in 1973. Since then we have evaluated 30 cases from the midwest region of the United States. We undertook a study to evaluate possible distinguishing features between cases of moyamoya disease in the midwestern United States and prototypical cases from southeast Asia.

\section{CLINICAL MATERIAL AND METHODS}

We identified patients with moyamoya disease who underwent evaluation at the University of Iowa using two search techniques. Specifically, we queried the University of Iowa Stroke Database to determine all patients with cerebrovascular events who were admitted to the institution since January 1973 and the Medical Records Department database in which categorization of moyamoya disease as a diagnostic entity began in January 1979. Our search of these databases tracked patient records through December 31, 1997.

Each case was categorized as classic moyamoya disease, probable moyamoya disease, or akin moyamoya disease, according to the definitions proposed by Fukui.[2] Cases were analyzed demographically (age at diagnosis, sex) and clinically (presenting symptoms, treatment). We reviewed patient charts for demographic characteristics, clinical presentation, and treatment methods. We then compared the data gathered at the University of Iowa with other case series reports from southeast Asia 
and the United States to evaluate disease similarities.

\section{RESULTS}

Since 1973, there have been 30 patients diagnosed with moyamoya disease at the University of Iowa. Of these cases, most (26 patients) fit the classic definition, with only three cases of probable moyamoya disease and one case of akin moyamoya disease (Down's syndrome). The demographic breakdown was similar to that reported previously in United States populations. Most patients in the Midwest were between the ages of 30 and 50 years at the time of diagnosis, but there was a small cluster of four patients younger than 16 years of age. There were 22 women and eight men (ratio 2.4:1) overall. A similar bimodal age distribution and female preponderance was seen in our patients as in other studies (Fig. 1).

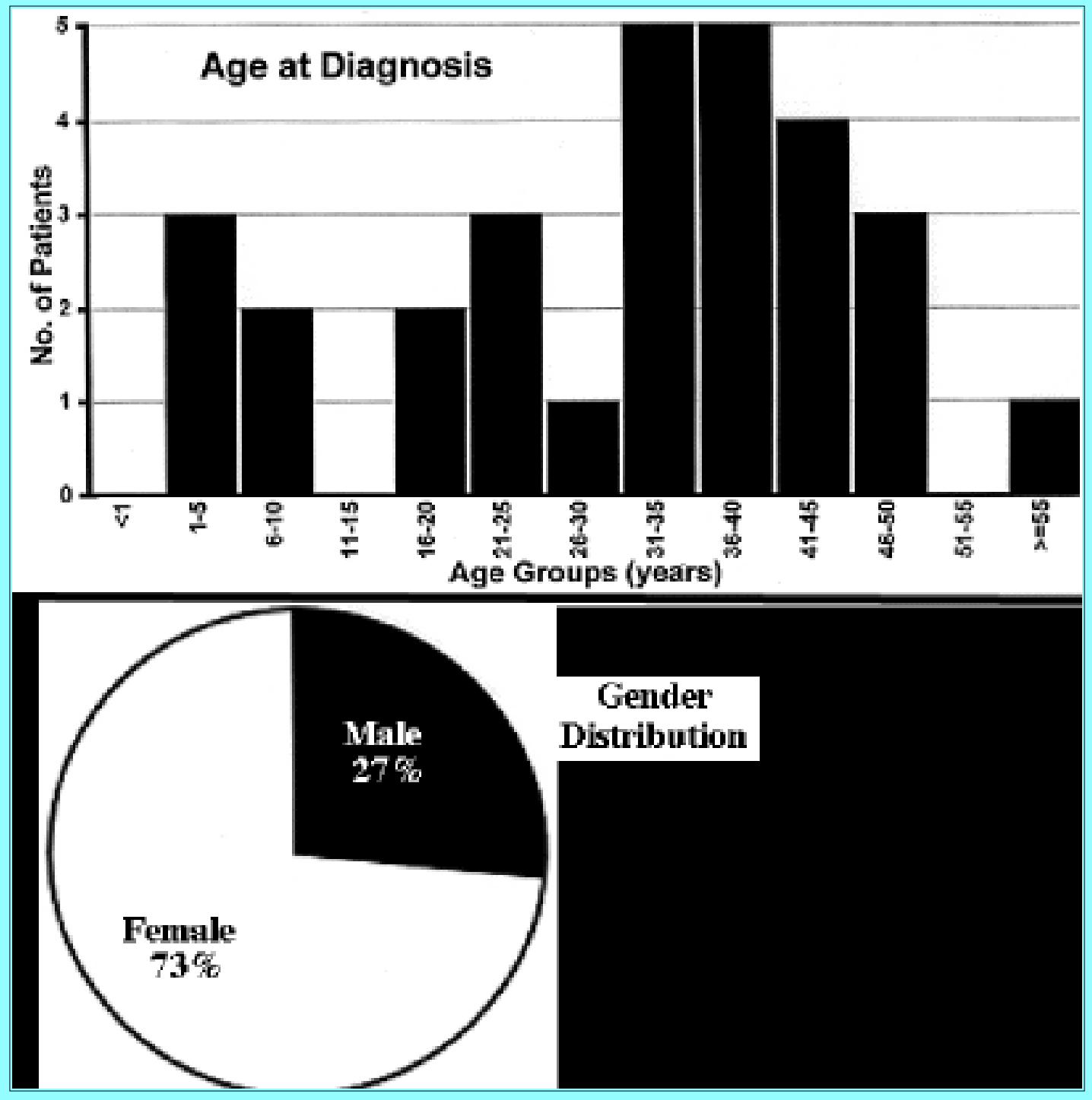

Fig. 1. Demographic data obtained at the time of diagnosis. Upper: Bar graph showing the distribution according to age in all patients with moyamoya disease. The typical bimodal presentation during the first and fourth decades is apparent. Lower: Pie chart showing the gender distribution in our population.

Of the pediatric cases, three presented with clear evidence of ischemia; none showed evidence of hemorrhage. Ischemia was also the leading cause for presentation among adults (80\%); however, three adults (12\%) presented with hemorrhage and two (8\%) with seizures (Fig. 2). 


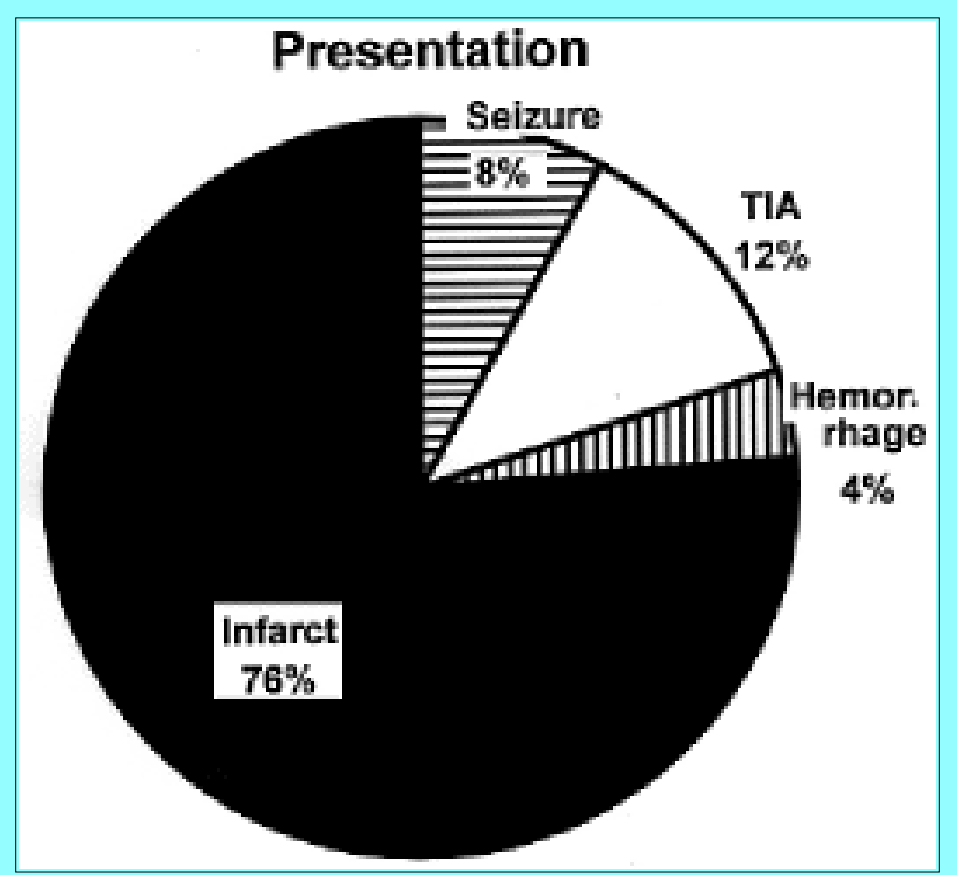

Fig. 2. Pie chart depicting breakdown of presenting symptoms, which indicates that patients generally present with ischemic complications.

Of the total population, 14 patients $(46 \%)$ underwent surgery. Seven patients underwent superficial temporal artery to middle cerebral artery anastomosis (three bilateral) and seven underwent a synangiosis procedure (either encephaloduroarteriosynangiosis or encephalomyosynangiosis). Three of the patients in the synangiosis groups also underwent bilateral procedures. The remaining 16 patients underwent medical therapy with warfarin, aspirin, ticlopidine, or subcutaneous heparin.

\section{DISCUSSION}

\section{Epidemiology of Moyamoya Disease in the Midwest United States and Southeast Asia}

The largest study to date on the epidemiological and radiological features of moyamoya disease in the United States was published by Numaguchi, et al.,[12] in 1997. The authors found 88 previously reported cases in the United States; in their critical analysis, however, they found that only 20 of these cases could be definitely considered moyamoya disease. They were able to collect an additional 26 unpublished cases from eight United States medical centers for a total of 46 definite cases of moyamoya disease in the United States. The authors reported that women have a predilection for the disease (71\%), which is similar to results reported in southeast Asian literature as well as those obtained the current report. The age range of these 98 patients was similar to the patients in our series (6 months-67 years vs. 18 months-49 years, respectively).

In the large epidemiological surveys undertaken in Japan, it was estimated that the number of patients treated was 3900 in 1994, which is slightly higher than the estimate of 3300 reported in the 1989 survey.[14] Analysis of the data in these studies showed that the disease predominates in the younger age groups, with a female to male ratio of 1.7:1. The overall incidence of moyamoya disease in Japan is one patient per 1,000,000, and 100 new cases are diagnosed each year. It is difficult to predict the prevalence and incidence in the United States because referral patterns cannot be known with certainty. A rough estimate can be formed based on Iowa's 2.5 million population. If all cases from the state were referred to the University of Iowa, this would yield a prevalence of 1.2 and an incidence of 0.052 per 100,000 persons. These are both lower than the 3.16 and 0.35 prevalence and incidence rates, respectively, 
reported from Japan but higher than previously estimated.

The commonly reported clinical manifestations of moyamoya disease reported in this study include ischemia (transient ischemic attacks [TIAs], reversible ischemic neurological deficits, and strokes), hemorrhage, headache, and seizure. The study reported by Yamaguchi, et al.,[18] in 1983 found 591 definite cases of moyamoya disease. The rate of hemorrhage in this series among men was lower than among women ( $29.6 \%$ vs. $39.1 \%$, respectively). When the rates of TIA and stroke were combined, ischemic symptoms were more frequent than hemorrhage among men $(45.9 \%)$ but were nearly equal to hemorrhage in women (38.1\%). These data are similar to those reported in the series by Handa, et al.,[5] Ohno, et al.,[13] Kudu,[7] and the cooperative studies in Japan from 1974 to 1984. Nishimoto and Takeuchi,[10] described 389 patients with definite moyamoya disease in 1981. Ischemic symptoms were more common than hemorrhage in these patients as well, but there was a predilection for hemorrhage in patients greater than the age of 15 years ( $65 \%$ vs. $5 \%$, respectively). The total number of pediatric patients who presented with ischemic symptoms of TIA and stroke was $122(78 \%)$, whereas among adults it was $58(24 \%)$.

The signs and symptoms most frequently observed in children in the studies reported from Japan are TIAs with hemiparesis, monoparesis, or sensory impairment. They occur bilaterally or on alternating sides because both cerebral hemispheres are affected. Adults most often presented with intracranial hemorrhage caused by intraventricular, subarachnoid, or intraparenchymal bleeding. Unfortunately, the prognosis in the patients who present with hemorrhage is usually much worse than those who present with ischemic symptoms.

In the United States, the clinical presentation may have different demographics. Chiu and colleagues[1] from the University of Texas Health Sciences Center at Houston reported on 32 cases of definite moyamoya disease. Among adults, 23 patients (85\%) presented with ischemic symptoms (TIA and stroke) and three presented with intracranial hemorrhage. The ethnic background of this patient population in Houston, Texas, was 63\% Caucasian, 17\% African American, 14\% Hispanic, and 6\% Asian. Numaguchi, et al.,[12] reported a similar low rate of hemorrhage (14\%) among adults in their United States multicenter study. This is supported by rates of hemorrhage (29\%) that are higher in United States populations comprised of a higher percentage of Asian patients such as in Hawaii.[4] Thus, the clinical presentation of the disease may vary with the ethnicity of the population studied, and there may be genetic and/or environmental cofactors that affect disease expression among different patient groups.

Historically, the clinical presentation of patients with moyamoya disease indicates that ischemic episodes are more common in younger patients and that hemorrhagic presentations are more common in older patients, in most series reported from Japan. Other presenting symptoms include seizures, sensory attacks, headache, and visual disturbances. The differences in presentation between older and younger patients remains speculative, but the occurrence of hemorrhage is considered to be due to the effects of aging and hypertensive loading that cause formation of microaneurysms along the vessel wall.[9] These abnormally developed networks, created as a consequence of the occlusion of normal arterial channels, result in rupture of the basal moyamoya vessels. Alternatively, the ischemic consequences of moyamoya disease are thought to be due to failed compensation of cerebral blood flow through collateral vessels that are normally supplied by branches of the circle of Willis. Why one type of compensatory pathophysiological manifestation develops and not another in different individuals is unknown.

The relative preponderance of ischemic symptoms in our adult patients may reflect a disease with 
expression based on multiple genetic and or environmental factors. It may also reflect a detection bias because more angiographic studies were performed in those patients who presented with stroke difference than in those who sustained ischemic episodes. The relatively few children evaluated in this series may also reflect an inadequate index of suspicion of moyamoya as a cause of stroke in younger patients.

\section{References}

1. Chui D, Shedden B, Bratina P, et al: Clinical features of moyamoya disease in the U.S. Stroke 29:1347-1351, 1998

2. Fukui M: Current state of study on moyamoya disease in Japan. Surg Neurol 47:138-143, 1997

3. Goto F, Yonekawa Y: Worldwide distribution of moyamoya disease. Neurol Med Chir 32:883-886, 1992

4. Graham JF, Matoba A: A survey of moyamoya disease in Hawaii. Clin Neurol Neurosurg 99 (Suppl 2):S31-S35, 1997

5. Handa H, Yonekawa Y, Goto Y, et al: Filing of 1500 cases of spontaneous occlusion of the circle of Willis, in Research Committee on Spontaneous Occlusion of the Circle of Willis ("Moyamoya Disease") (eds): Annual Report 1984. Tokyo: Ministry of Health and Welfare, 1985, pp 14-22

6. Kudo T: Occlusion of the internal carotid artery and the type of recovery of cerebral blood circulation. Clin Neurol 1:199-200, 1960

7. Kudo T: [Spontaneous occlusion of the circle of Willis.] Neurol Surg 3:711-724, 1975 (Jpn)

8. Kudo T: Spontaneous occlusion of the circle of Willis: a disease apparently confined to Japanese. Neurology 18:485-496, 1968

9. Matsushima M. Moyamoya disease, in Youmans JR (ed): Neurological Surgery, ed 4. Philadelphia: WB Saunders, 1996, pp 1202-1223

10. Nishimoto A, Takeuchi T: Abnormal cerebrovascular network related to the internal carotid arteries. J Neurosurg 29:255-260, 1968

11. Nishimoto A, Ueta K, Onbe H: Cooperative study on moyamoya disease in Japan, in Abstracts of the 10th Meeting on Surgery for Stroke. Tokyo: Nyuuron-sha, 1981, pp 53-58

12. Numaguchi Y, Gonzalez CF, Davis PC, et al: Moyamoya disease in the United States. Clin Neurol Neurosurg 99 (Suppl 2):S26-S30, 1997

13. Ohno Y, Yonekawa Y, Handa H, et al: Epidemiological features of spontaneous occlusion of the circle of Willis--results of the cooperative study, in Research Committee on Epidemiology of Intractable Diseases (eds): Annual Report 1986. 1987, pp 29-32 [Ref. unverified]

14. Research Committee on Epidemiology of Intractable Diseases (eds): Annual Report. Tochigi, Japan, 1991 [Ref. unverified]

15. Takeuchi K: Carotid artery obstruction. Shinkei Shimpo 5:511-543, 1961 
16. Takeuchi K, Shimuzu K: Hypogenesis of bilateral internal carotid arteries. No To Shinkei 9:37-43, 1957

17. Wakai K, Tamakoshi A, Ikezaki K, et al: Epidemiological features of Moyamoya disease in Japan: findings from a nationwide survey. Clin Neurol Neurosurg 99 (Suppl 2):S1-S5, 1997

18. Yamaguchi T, Tsashiro M, Hasegawa Y: All cases of occlusion of the circle of Willis collected in the cooperative study in Japan. Summary of six years, in Research Committee on Spontaneous Occlusion of the Circle of Willis ("Moyamoya Disease") (eds): Annual Report 1982. Tokyo: Ministry of Health and Welfare, 1983, pp 15-27

19. Yonekawa Y, Handa H, Okuno T: Moyamoya disease: diagnosis, treatment, and recent achievements. Stroke 1:805-829, 1986

Manuscript received September 15, 1998.

Accepted in final form October 23, 1998.

Address reprint requests to: P. Charles Garrell, M.D., Division of Neurosurgery, University of Iowa Hospitals and Clinics, Iowa City, Iowa 52242. email: V420@UIHCjes2.UIHC.uiowa.edu. 